03

\title{
Генерация предельно-коротких импульсов при возбуждении нелинейной резонансной среды световым зайчиком, движущимся со сверхсветовой скоростью
}

\author{
(C) Р.М. Архипов ${ }^{1,2}$, Д.О. Жигулева ${ }^{1,3}$, А.В. Пахомов ${ }^{2}$, М.В. Архипов ${ }^{1}$, И. Бабушкин ${ }^{5,6}$, Н.Н. Розанов $^{2,4,7}$ \\ ${ }^{1}$ Санкт-Петербургский государственный университет, \\ 199034 Санкт-Петербург, Россия \\ 2 Университет ИТМО, \\ 197101 Санкт-Петербург, Россия \\ ${ }^{3}$ Heinrich-Heine-Universität Düsseldorf, \\ 40225 Düsseldorf, Germany \\ ${ }^{4}$ Государственный оптический институт им. С.И. Вавилова, \\ 199053 Санкт-Петербург, Россия \\ ${ }^{5}$ Institute of Quantum Optics, Leibniz University Hannover, \\ 30167 Hannover, Germany \\ ${ }^{6}$ Max Born Institute, \\ Berlin, 12489 Germany \\ ${ }^{7}$ Физико-технический институт им. А.Ф. Иофрфе, \\ 194021 Санкт-Петербург, Россия \\ e-mail: arkhipovrostislav@gmail.com
}

Поступила в редакцию 01.12.2017 г.

Теоретически проанализирована возможность генерации квазиуниполярных предельно-коротких импульсов (длительностью в несколько периодов колебаний поля) при возбуждении кольцевой, нелинейной, резонасной среды световым ,зайчиком“. При этом зайчик вращается по кругу, вдоль которого распределены частицы среды, со скоростью, превосходящей скорость света в пустоте. Показано, что путем введения в систему задержки в виде фазовой пластинки возможно управлять формой, длительностью и амплитудой генерируемых импульсов.

DOI: $10.21883 /$ OS.2018.04.45752.278-17

\section{Введение}

Излучение равномерно движущихся источников, скорость которых превосходит фазовую скорость света в среде или в вакууме, уже давно привлекает внимание различных исследователей. Возможные реализации и примеры таких источников рассмотрены в классических обзорах Б.М. Болотовского и В.Л. Гинзбурга $[1,2]$, см. также [3-6]. К примерам таких источников можно отнести область пересечения волнового фронта с плоским экраном при наклонном падении короткого импульса на экран $[1,3]$, световое пятно („зайчик“) на удаленном экране от равномерно вращающегося источника [1-3], заряженную частицу, движущуюся со скоростью, большей фазовой скорости света в среде и являющуюся источником излучения Вавилова-Черенкова [7-11], максимум светового импульса, распространяющегося в нелинейной усиливающей среде [12-14], а также оптические солитоны [15] и др.

В последнее время огромный интерес вызывает генерация предельно-коротких импульсов, длительность которых составляет порядка периода колебаний световой волны и меньше [16-18]. Такие импульсы открыли возможность изучать и управлять динамикой сверхбыстрых процессов в веществе. Например, они позволяют осу- ществлять контроль динамики волновых пакетов в атомах, молекулах, твердых телах и наноструктурах [19-22]. Обычные генерируемые импульсы являются биполярными, т. е. электрическая площадь таких импульсов (интеграл от вектора напряженности электрического поля по времени в заданной точке пространства [23]) равен 0. Недавно появился ряд работ, в которых изучается возможность генерации униполярных импульсов света (т. е. импульсов, электрическая площадь которых может быть отлична от 0) при сверхсветовом возбуждении нелинейных сред, см. [24-28], а также обзоры [29,30] и ссылки в них. Получить полностью униполярные импульсы (УИ) трудно, так как ускорение связанной системы зарядов всегда является биполярным. Однако в ряде ситуаций удается получить квазиуниполярные импульсы (КУИ), содержащие „всплеск“ электрического поля и длинный хвост противоположной полярности, но меньшей амплитуды. Очевидно, что такие импульсы представляют несомненный интерес ввиду возможности однонаправленного воздействия на электрические заряды и, следовательно, перспективным является возможность применения таких импульсов для контроля динамики волновых пакетов вещества [16-21,29-30] и сверхбыстрого управления параметров резонансных сред, в частности решетками поляризации и разности заселенностей [31-34]. 
В работах [24-28] показана возможность генерации КУИ при сверхсветовом возбуждении нелинейных сред с различной геометрией (прямолинейной [24] и круговой [25]) несколькими световыми зайчиками. В работах $[26,27]$ проведено общее рассмотрение возможности генерации КУИ для случая сред, обладающих произвольной нелинейной связью с полем. В работе [28] предсказана возможность генерации субциклового импульса при отражении одноциклового импульса от тонкой металлической или диэлектрической пленки в одномерном случае.

В настоящей работе анализируется еще одна удивительная возможность генерации КУИ. Источником в данном случае является один сверхсветовой зайчик, вращающийся со сверхсветовой скоростью по кругу, вдоль которого распределены резонансные атомы нелинейной среды. Отметим, что в [25] рассматривалась возможность генерации КУИ в случае кольцевой геометрии комбинационно-активной среды (КАС), однако среда в этом случае возбуждалась одновременно двумя световыми зайчиками. Ниже будет рассмотрена еще одна, на наш взгляд, более простая возможность генерации КУИ в случае, когда один световой зайчик вращается со сверхсветовой скоростью по окружности, вдоль которой распределена нелинейная среда. Отличительной особенностью предложенного метода от расмотренных авторами ранее также является то, что в систему вводится долнительная задержка в виде фазовой пластинки, что позволяет управлять амплитудой и длительностью генерируемых импульсов.

\section{Теоретическая модель и рассмативаемая система}

Для простоты будем считать, что среда состоит из нелинейных осцилляторов с частотой собственных колебаний $\omega_{0}$, расположенных на окружности радиуса $R$ (рис. 1). Как и в [27], будем считать, что среда обладает квадратично-нелинейной связью с полем $E$, т.е. эффективное значение поляризации среды описывается уравнением следующего вида $[27,29,35,36]$ :

$$
\ddot{P}+\gamma \dot{P}+\omega_{0}^{2} P=g_{0} E^{2} .
$$

Наиболее простым физическим примером подобной среды являются комбинационно-активные среды (КАС), для которых вследствие наличия в среде двух связанных осцилляторов электронного (высокочастотного) и низкочастотного (ядерного) с сильно отличающимися резонансными частотами возможно исключить из уравнений движение высокочастотного осциллятора при действии импульса оптического диапазона частот. Это дает для эффективного отклика среды уравнение (1) с квадратом действующего электрического поля в правой части [27-29]. Однако важно отметить, что подобные нелинейно-связанные резонансы также могут быть реализованы в различных типах наночастиц, таких как

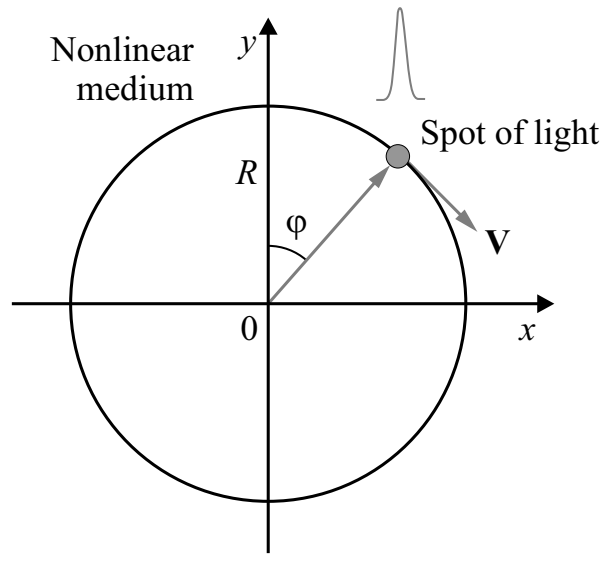

Рис. 1. Кольцевая геометрия резонансной среды, возбуждаемой световым пятном (сплошной кружок), движущимся со скоростью $V$ вдоль круга. Излучение среды регистрируется в центре круга $(\mathrm{O})$.

связанные полупроводниковые квантовые точки, микрои нанорезонаторы, плазмонные резонансы в металлических наноантеннах ит.п., что делает возможным использование уравнений типа (1) для описания оптического отклика большого числа наноструктурированных сред [37-39]. Рассматриваемая на рис. 1 кольцевая геометрия задачи может быть аналогична кольцевым массивам наночастиц, свойства которых и взаимодействие с электромагнитным излучением также изучаются в последнее время [40-43].

Обычно комбинационно-активные молекулы являются анизотропными, поэтому в рассматриваемой схеме необходимо, чтобы оси частиц были ориентированы перпендикулярно плоскости окружности в каждой точке на круге и возбуждающее поле было поляризовано также в этом направлении.

Предполагаем, как и в работе [27], что падающий импульс имеет длительность много меньше периода собственных колебаний среды. При данном предположении решение уравнения (1) может быть представлено в виде [27]:

$$
P(t)=\Pi_{1} \sin \omega_{0} t
$$

где $\Pi_{1} \equiv \frac{g_{0}}{\omega_{0}} \int_{-\infty}^{\infty} E^{2}(t) \cos \left(\omega_{0} t\right) d t$.

Основная идея метода получения униполярных импульсов заключается в следующем [24,29]. Если среда освещается последовательностью коротких импульсов, следующих с периодом, равным половине периода колебаний осцилляторов среды $T_{0} / 2\left(\omega_{0}=2 \pi / T_{0}\right)$, то первый импульс вызывает колебания осциллятора на собственной частоте, тогда как второй импульс останавливает их (рис. 2). В результате смещение осциллятора имеет вид полуволны колебаний (которая, согласно (2), имеет вид половины синусоиды), которая схематически изображена на рис. 2. 


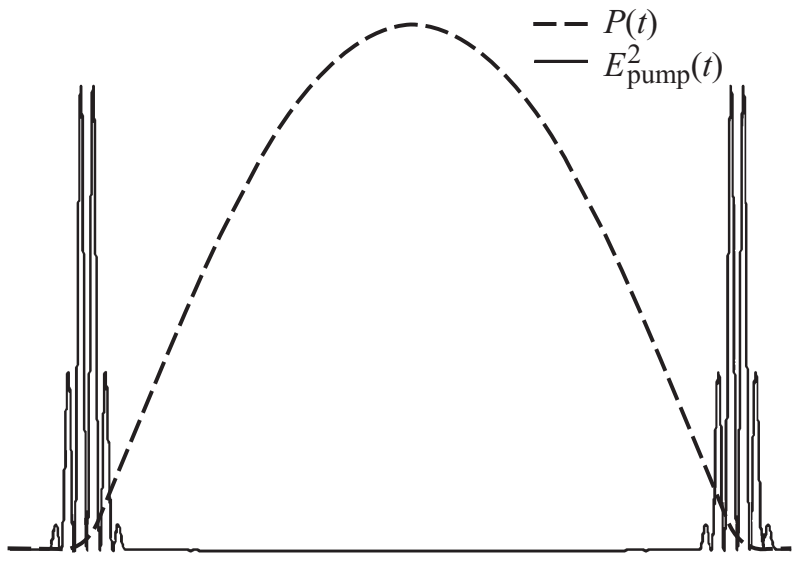

Рис. 2. Поляризация среды $P(t)$ (штриховая линия) - полуволна синусоиды и квадрат поля возбуждения накачки $E_{\text {pump }}^{2}(t)$ (сплошная линия) при возбуждении нелинейного осциллятора последовательностью из двух импульсов с периодом $T_{p}=T_{0} / 2$. Иллюстрация носит схематичный характер.

Предположим теперь, что осцилляторы возбуждаются одним световым пятном (световой зайчик), бегущим по кругу с постоянной сверхсветовой линейной скоростью $V>c$. Возбуждающий импульс дважды проходит круг, после чего выключается. Затухание осцилляторов за время действия возбуждения считается пренебрежимо слабым.

Вторичное излучение осцилляторов детектируется в центре круга (рис. 1) на большом расстоянии от осцилляторов после временной задержки, линейно зависящей от полярного угла $\varphi$. Такая задержка может быть реализована, например, путем помещения фазовой пластинки, толщина которой линейно нарастает с углом $\varphi$ [44]; ее можно представить как прозрачный транспарант, время прохождения которого определяется формулой

$$
\tau(\varphi)=\tau_{0}\left(1+A_{m} \varphi\right),
$$

где $A_{m}$ - некоторая амплитуда модуляции.

Поле излучения среды в центре круга в дальней зоне представляет собой сумму сигналов в виде половин синусоид (см. формулу (2) и рис. 2) от отдельных осцилляторов с определенной задержкой друг относительно друга. Оно записывается в виде [26,27]

$$
E(t) \sim \int_{0}^{2 \pi} \sum_{k=1}^{2} \sin \left[\omega_{0} t_{k}(\varphi)\right] \Theta\left(t_{k}(\varphi)\right) d \varphi .
$$

Здесь

$$
t_{k}(\varphi)=t-\frac{R}{V} \varphi-\tau(\varphi)-(k-1) T_{p},
$$

$k$ имеет смысл номера прохода зайчика по окружности, член $R \varphi / V$ - время возбуждения осциллятора с угловой координатой $\varphi, T_{p}=T_{0} / 2, \Theta-$ ступенчатая функция Хевисайда. Эти выражения описывают возбуждение осцилляторов на первом обороте зайчика по окружности и остановку этих осцилляций на втором обороте, как это указывалось выше (рис. 2). Функция синус в подынтегральном выражении появляется из-за того, что, согласно формуле (2), поляризация среды может быть описана синусоидальным сигналом. Излучаемое поле, пропорциональное второй производной амплитуды (ускорению зарядов), будет содержать высокочастотные осцилляции (ВЧ) в моменты запуска и остановки колебаний. Поэтому получаемый импульс не будет строго униполярным. Однако высокочастотные компоненты могут быть погашены фильтром [24-27], что позволяет нам не учитывать их в дальнейшем.

Вместо схемы с транспарантом, обладающим непрерывной угловой зависимостью фазового набега, можно представить себе схему с дискретно расположенными по окружности осцилляторами. Если угловой период их размещения выбрать в соответствии с условием $T_{p}=T_{0} / 2$, образовавшаяся дискретная система будет аналогичной рассмотренной выше непрерывной, но центр окружности может быть связан с каждым из осцилляторов сектором прозрачного вещества с соответствующим показателем преломления. Вместо секторов могут применяться световоды разной оптической длины, как изготовленные из веществ с разным показателем преломления, так и имеющие различную геометрическую длину. Схема может быть реализована и с помощью фотонно-кристаллических световодов, параметры которых (дисперсия, пропускание) могут легко варьироваться в широких пределах [45]. Линейную нарастающую с углом задержку можно также реализовать, если разместить осцилляторы на витки спирали Архимеда. В этом случае параметр $A_{m}$ имеет смысл шага спирали Архимеда.

Отметим, что быстрое вращение светового пятнышка по кругу может быть реализовано с помощью дефлекторов лазерного излучения, позволяющих быстро изменять направление распространения лазерного пучка в пространстве, см. обзор [46] и работу [47].

\section{Результаты численного моделирования и обсуждение результатов}

На рис. 3 представлены результаты вычислений по формулам (3)-(5) при $V / c=4, \quad \tau_{0} c / R=1 \quad$ и $T_{p}=2 \pi R / V=T_{0} / 2$ в отсутствие $\left(A_{m}=0\right.$, рис. $\left.3, a\right)$ и при наличии $\left(A_{m}=0.5\right.$, рис. $\left.3, b\right)$ задержки. При $A_{m}=0$ сигнал в центре окружности имеет форму одного периода колебания осциллятора, а приближенно параболическое нарастание амплитуды от нулевого значения связано с суммированием начальных линейно нарастающих компонентов полупериода характеристического колебания осцилляторов, задержанных во времени.

При введении задержки полуволны от отдельных участков среды приходят в центр круга с запаздыванием и, как и в [24-27], происходит генерация КУИ с плоской вершиной. Очевидно, что длительностью и амплитудой 

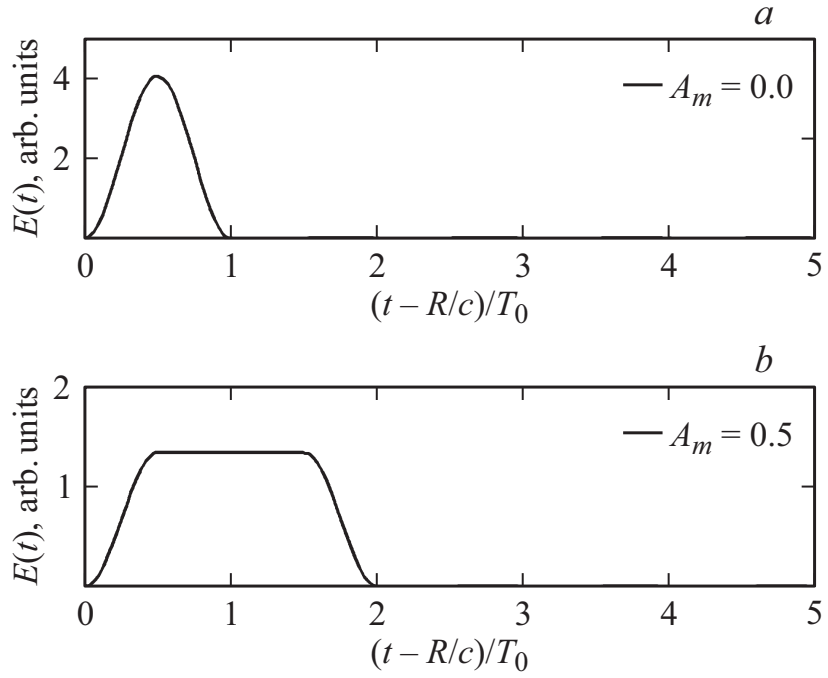

Рис. 3. Форма КУИ в центре круга при различных параметрах „эффективной задержки“: $A_{m}=0(a)$ и $A_{m}=0.5(b)$.

получаемых импульсов можно управлять, изменяя параметр задержки $A_{m}$.

Типичная величина частоты собственных колебаний осцилляторов может составлять по порядку величины $\omega_{0} / 2 \pi \simeq 10^{12} \div 10^{14} \mathrm{~Hz}$ (например, частоты колебаний различных молекул), т.е. лежит в терагерцовом и инфракрасном (ИК) диапазоне. Поэтому генерируемое излучение может лежать в терагерцовой или ИК области спектра. Таким образом, предложенный метод может служить еще одним методом генерации $\mathrm{THz}$ излучения, способы получения которого активно изучаются на сегодняшний день [48-51].

Результаты численных расчетов, проведенных на основе прямого численного интегрирования системы уравнений Максвелла-Блоха, показывают, что амплитуда генерируемого импульса по порядку величины может быть в 10 раз меньше амплитуды падающего импульса [28].

Еще раз оговорим, что при указанной выше фильтрации ВЧ колебаний осцилляторов, возникающих в моменты их старта и остановки, получаемые импульсы, вообще говоря, не являются строго униполярными, a могут содержать длинный хвост противоположной полярности, форма которого зависит от характеристик фильтра.

Выше предполагалось, что длительность импульса возбуждения меньше времени затухания колебаний осцилляторов среды. Следовательно, взаимодействие импульса со средой можно считать когерентным. Поэтому проанализированный метод генерации КУИ может рассматриваться как еще одно из устройств нелинейной фотоники на базе когерентного взаимодействия света с веществом („,coherent photonics devices“), примеры которых рассмотрены в обзоре [31].

Рассмотренный способ получения КУИ с помощью сверхсветового зайчика представляет собой пример сверхсветовых источников электромагнитного излучения, в дополнение к рассмотренным ранее в [1-3].

\section{Заключение}

Таким образом, в работе предложена и теоретически проанализирована возможность получения квазиуниполярных импульсов при возбуждении сверхсветовым зайчиком расположенных вдоль кольца нелинейных осцилляторов. Показано, что путем введения эффективной задержки (фазовой пластинки) в распространении вторичных волн от осцилляторов в точку наблюдения достигается возможность генерации квазиуниполярных, предельно коротких, приближенно прямоугольных видеоимпульсов. Длительность генерируемых импульсов составляет несколько периодов колебаний осцилляторов среды и может лежать в $\mathrm{THz}$ диапазоне.

Результаты численного моделирования показали, что длительность импульсов и их амплитуда могут варьироваться в широких пределах с помощью изменения параметров задержки. Предложенный в работе метод генерации предельно коротких импульсов интересен тем, что выбор сред с определенной резонансной частотой позволяет получать такие импульсы в различных спектральных интервалах, например, в терагерцовой или ИК области.

Исследование сверхсветового возбуждения кольцевой нелинейной среды световым зайчиком, вращающимся по кругу, частично поддержано грантом РФФИ 16-02-00762а. Исследование возможности генерации квазиуниполярных предельно-коротких импульсов выполнено за счет гранта Российского научного фонда (проект № 17-19-01097).

\section{Список литературы}

[1] Болотовский Б.М., Гинзбург В.Л. // УФН. 1972. Т. 106. № 4. C. 577; Bolotovskii B.M., Ginzburg V.L. // Sov. Phys. Usp. 1972. V. 15. N 2. P. 184.

[2] Болотовский Б.М., Серов А.В. // УФН. 2005. Т. 175. № 9. C. 943; Bolotovskii B.M., Serov A.V. // Phys. Usp. 2005. V. 48. N 9. P. 903.

[3] Гинзбург В.Л. Теоретическая физика и астрофизика. Дополнительные главы (2-е издание). Рипол Классик, 1981. Гл. 9. С. 211; Ginzburg V.L. Theoretical physics and astrophysics. Pergamon Press Ltd, 1979. Chapter VIII. P. 171.

[4] Малыкин Г.Б., Романец, Е.А. // Опт. и спектр. 2012. Т. 112. № 6. C. 993; Malykin G.B., Romanets E.A. // Opt. Spectr. 2012. V. 112. N 6. P. 920.

[5] Recami E. // Foundations of Physics. 2001. T. 31. N 7. C. 1119.

[6] Recami E., Fontana F., Garavaglia R. // Int. J. Mod. Phys. A. 2000. V. 15. P. 2793.

[7] Черенков П.А. // ДАН СССР. 1934. Т. 2. № 8. С. 451.

[8] Cerenkov P.A. // Phys. Rev. 1937. V. 52. P. 378.

[9] Тамм И.Е., Франк И.М. // ДАН СССР. 1937. Т. 14. С. 107.

[10] Франк И.М. // УФН. 1982. Т. 143. № 1. С. 111; Frank I.M. // Sov. Phys. Usp. 1984. V. 27. P. 385. 
[11] Болотовский Б.М. // УФН. 2009. Т. 179. № 11. С. 1161; Bolotovskii B.M. // Phys. Usp. 2009. V. 52. P. 1099.

[12] Басов Н.Г., Амбариумян Р.В., Зуев В.С., Крюков П.Г., Летохов В.С. // ЖЭТФ. 1966. Т. 50. № 1. С. 23; Basov N.G., Ambartsumyan R.V., Zuev V.S., Kryukov P.G., Letokhov V.S. // Sov. Phys. JETP. 1966. V. 23. N 1. P. 16.

[13] Крюков П.Г., Летохов В.С. // УФН. 1969. Т. 99. № 2. C. 169; Kryukov P.G., Letokhov V.S. // Sov. Phys. Usp. 1970. V. 12. P. 641.

[14] Ораевский А.Н. // УФН. 1998. Т. 168. № 12. С. 1311; Oraevskii A.N. // Phys. Usp. 1998. V. 41. P. 1199.

[15] Skryabin D.V., Gorbach A.V. // Rev. Mod. Phys. 2010. V. 82. P. 1287.

[16] Krausz F., Ivanov M. // Rev. Mod. Phys. 2009. V. 81. P. 163.

[17] Manzoni C., Mücke O.D., Cirmi G., Fang S., Moses J., Huang S.-W., Hong K.-H., Cerullo G., Kärtner F.X. // Laser Photonics Rev. 2015. V .9. P. 129.

[18] Calegari F., Sansone G., Stagira S., Vozzi C., Nisoli M. // J. Phys. B: At. Mol. Opt. Phys. 2016. V. 49. P. 062001.

[19] Gallmann L., Cirelli C., Keller U. // Ann. Rev. Phys. Chem. 2012. V. 63. P. 447.

[20] Ramasesha K., Leone S.R., Neumark D.M. // Annu. Rev. Phys. Chem. 2016. V. 67. P. 41.

[21] Ciappina M.F., Pérez-Hernández J.A., Landsman A.S. // Rep. Prog. Phys. 2017. V. 80. P. 054401.

[22] Kozak M., McNeur J., Leedle K.J., Deng H., Schonenberger N., Ruehl A., Hartl I., Harris J.S., Byer R.L., Hommelhoff P. // Nat. Commun. 2017. V. 8. P. 1.

[23] Розанов Н.Н. // Опт. спектр. 2009. Т. 107. № 5. С. 761; Rosanov N.N. // Opt. Spectr. 2009. V. 107. N 5. P. 721.

[24] Arkhipov R.M., Arkhipov M.V., Belov P.A., Tolmachev Yu.A., Babushkin I. // Las. Phys. Lett. 2016. V. 13. N 4. P. 046001.

[25] Arkhipov R.M., Pakhomov A.V, Babushkin I.V., Arkhipov M.V., Tolmachev Yu.A., Rosanov N.N. // J. Opt. Soc. Am. B. 2016. V. 33. N 12. P. 2518.

[26] Pakhomov A.V., Arkhipov R.M., Babushkin I.V., Arkhipov M.V., Rosanov N.N. // Laser Phys. Lett. 2016. V. 13. P. 126001.

[27] Pakhomov A.V., Arkhipov R.M., Babushkin I.V., Arkhipov M.V., Tolmachev Yu.A., Rosanov N.N. // Phys. Rev. A. 2017. V. 95. P. 013804.

[28] Arkhipov M.V., Arkhipov R.M., Pakhomov A.V., Babushkin I., Demircan A., Morgner U., Rosanov N.N. // Opt. Lett. 2017. V. 42. N 11. P. 1.

[29] Архипов Р.М., Пахомов А.В., Архипов М.В., Бабушкин И., Толмачев Ю.А., Розанов Н.Н. // Письма в ЖЭТФ. 2017. T. 105. № 6. C. 388; Arkhipov R.M., Pakhomov A.V., Arkhipov M.V., Babushkin I., Tolmachev Yu.A., Rosanov N.N. // JETP Lett. 2017. V. 105. N 6. P. 408.

[30] Arkhipov R.M., Pakhomov A.V., Arkhipov M.V., Babushkin I., Tolmachev Yu.A., Rosanov N.N. // Laser Phys. 2017. V. 27. N 5. P. 053001.

[31] Архипов Р.М., Архипов М.В., Пахомов А.В., Бабушкин И., Розанов Н.Н. // Опт. и спектр. 2017. Т. 122. № 6. С. 993; Arkhipov R.M., Arkhipov M.V., Pakhomov A.V., Babushkin I., Rosanov N.N. // Opt. Spectr. 2017. V. 122. N 6. C. 949.

[32] Архипов Р.М., Архипов М.В., Бабушкин И.В., Пахомов А.В., Розанов Н.Н. // Квант. Электр. 2017. Т. 47. № 7. C. 589; Arkhipov R.M., Arkhipov M.V., Babushkin I.V., Pakhomov A.V., Rosanov N.N. // Quantum Electron. 2017. V. 47. N 7. P. 589.
[33] Arkhipov R.M., Pakhomov A.V., Arkhipov M.V., Babushkin I., Rosanov N.N. // Laser Phys. Lett. 2017. V. 14. P. 095402.

[34] Arkhipov R.M., Pakhomov A.V., Arkhipov M.V., Babushkin I., Demircan A., Morgner U., Rosanov N.N. // Sci. Rep. 2017. V. 7. P. 12467.

[35] Ахманов С.А., Коротеев Н.И. Методы нелинейной оптики в спектроскопии рассеяния света: Активная спектроскопия рассеяния света. М.: Наука, 1981.

[36] Ахманов С.А., Никитин С.Ю. Физическая оптика. М.: Наука, 2004; Akhmanov S.A., Nikitin S.Y. Physical optics. Clarendon Press, 1997.

[37] Novotny L.N., van Hulst N. // Nat. Photon. 2011. V. 5. P. 83.

[38] Biagioni P., Huang J.-S., Hecht B. // Rep. Prog. Phys. 2012. V. 75. P. 024402.

[39] Mohan A., Felici M., Gallo P., Dwir B., Rudra A., Faist J., Kapon E. // Nat. Photon. 2010. V. 4. P. 302.

[40] Burin A.L. // Phys. Rev. A. 2006. V. 73. P. 066614.

[41] Fung K.H., Chan C.T. // Phys. Rev. B. 2008. V. 77. P. 205423.

[42] Fikioris G., Lygkouris S., Papakanellos P. // J. IEEE Trans. Antennas Propag. 2011. V. 59. P. 4615.

[43] Polishchuk I., Gozman M.I., Samoylova O.M., Burin A.L. // Phys. Lett. A. 2009. V. 373. P. 1396.

[44] Сойфер В.А. Дифракционная нанофотоника. М.: ФИЗМАТЛИТ, 2011. $680 \mathrm{c}$.

[45] Желтиков А.М. // УФН. 2007. Т. 177. С. 737; Zheltikov A.M. // Phys. Usp. 2007. V. 50. P. 705.

[46] Römer G.R.B.E., Bechtold P. // Phys. Procedia. 2014. V. 56. P. 29.

[47] Arkhipov R.M., Arkhipov M.V., Egorov V.S., Chekhonin I.A., Chekhonin M.A., Bagayev S.N. // J. Phys. 2015. V. 643. P. 012029.

[48] Roskos H.G., Thomson M.D., Kress M., Loeffler T. // Laser Photon. Rev. 2007. V. 1. P. 349.

[49] Reimann K. // Rep. Progr. Phys. 2007. V. 70. P. 1597.

[50] Bartel T., Gaal P., Reimann K., Woerner M., Elsaesser T. // Opt. Lett. 2005. V. 30. P. 2805.

[51] Babushkin I., Skupin S., Herrmann J. // Opt. Expr. 2010. V. 18. P. 9658. 